\title{
CELL-LIKE MAPS THAT ARE SHAPE EQUIVALENCES
}

\author{
JUNG-IN K. CHOI
}

(Communicated by Doug W. Curtis)

\begin{abstract}
Let $f: X^{\prime} \rightarrow X$ be a cell-like map between metric spaces and set $N_{f}=\left\{x \in X: f^{-1}(x) \neq\right.$ point $\}$. Even if $N_{f} \subset \bigcup_{n=1}^{\infty} B_{n}$, where each $B_{n}$ is closed and each $f \mid f^{-1}\left(B_{n}\right): f^{-1}\left(B_{n}\right) \rightarrow B_{n}$ is hereditary shape equivalence, $f$ may not be a hereditary shape equivalence. Conditions are placed on the $B_{n}$ 's to assure that $f$ is a hereditary shape equivalence. For example, if $N_{f} \subset$ $\bigcup_{n=1}^{\infty} B_{n}$, where $B_{n}$ is closed for each $n=1,2, \ldots, f \mid f^{-1}\left(B_{n}\right): f^{-1}\left(B_{n}\right) \rightarrow$ $B_{n}$ is a hereditary shape equivalence, and $B_{n}$ has arbitrary small neighborhoods whose boundaries miss $\bigcup_{i=1}^{\infty} B_{i}$, then $f$ is a hereditary shape equivalence. An immediate consequence is that if $\left\{B_{n}\right\}_{n=1}^{\infty}$ is a pairwise disjoint null-sequence and each $f \mid f^{-1}\left(B_{n}\right)$ is a hereditary shape equivalence, then $f$ is a hereditary shape equivalence. Previously G. Kozlowski showed that if $\left\{f^{-1}\left(B_{n}\right)\right\}_{n=1}^{\infty}$ is a pairwise disjoint null-sequence and each $f \mid f^{-1}\left(B_{n}\right)$ is a hereditary shape equivalence, then $f$ is a hereditary shape equivalence, which can be obtained as an immediate corollary of one of our results.
\end{abstract}

\section{INTRODUCTION}

For the most part cell-like maps behave in the expected fashion by being fine homotopy equivalences in the setting of ANR's and by being hereditary shape equivalences in the general setting of metric spaces (see [An, Ha, Ko, La, MR, Sh]). An exception is due to J. Taylor [Ta]. R. J. Daverman and J. J. Walsh [DW] modified Taylor's example [Ta] to obtain a cell-like map from a compactum with nontrivial shape onto the Hilbert cube such that the nondegeneracy set is contained in the countable union of finite dimensional compact sets. On the other hand, G. Kozlowski [Ko] proved that a cell-like map $f: X^{\prime} \rightarrow X$ from a compact ANR $X^{\prime}$ onto a metric space $X$ is a hereditary shape equivalence if there is a sequence $\left\{B_{n}\right\}_{n=1}^{\infty}$ of closed subsets of $X$ such that the nondegeneracy set is contained in $\bigcup_{n=1}^{\infty} B_{n}, f \mid f^{-1}\left(B_{n}\right)$ is a hereditary shape equivalence for each $n=1,2, \ldots$, and $\left\{f^{-1}\left(B_{n}\right)\right\}_{n=1}^{\infty}$ forms a pairwise disjoint null-sequence.

Received by the editors June 27, 1986 and, in revised form, November 17, 1986.

1980 Mathematics Subject Classification. Primary 55M15, 55P55; Secondary 54F40, 57N25.

Key words and phrases. Cell-like map, hereditary shape equivalence, ANR, slice-trivial, nondegeneracy set, null-sequence.

These results are from part of the author's Ph.D. dissertation completed under the direction of John J. Walsh at The University of Tennessee. 
Here we find sufficient conditions for cell-like maps to be hereditary shape equivalences and, furthermore, we extend the aforementioned result of G. Kozlowski's.

1. Definitions and notation. A cell-like map is a proper map with each pointinverse having trivial shape. A map $f: X \rightarrow Y$ is a hereditary shape equivalence provided $f \mid f^{-1}(A): f^{-1}(A) \rightarrow A$ is a shape equivalence for any closed subset $A$ of $Y$. It follows that a cell-like map is surjective (see Lemma 7 in [Ko]). For the fixed map $f: X^{\prime} \rightarrow X$ we introduce the following notation:

$$
\begin{aligned}
S^{\prime} & =f^{-1}(S) \text { for a subset } S \text { of } X, \\
\mathscr{U}^{\prime} & =\left\{f^{-1}(U) \mid U \in \mathscr{U}\right\} \text { for a cover } \mathscr{U} \text { of } X, \\
N_{f} & =\left\{x \mid f^{-1}(x) \neq \text { point }\right\} .
\end{aligned}
$$

For a subset $V$ of $X \times Y$ we adopt the following notation:

$$
\begin{aligned}
& \operatorname{dom} V=\{x \in X \mid(x, y) \in V \text { for some } y \in Y\}, \\
& V \mid K=\{(x, y) \mid(x, y) \in V \text { and } x \in K\} \text { for a subset } K \text { of } X, \\
& V|x=V|\{x\} \text { for } x \in X, \\
& V(x)=\{y \in Y \mid(x, y) \in V\} \text { for } x \in X .
\end{aligned}
$$

Suppose $U$ and $V$ are subsets of $X \times Y$ with $U \supset V$ and $g$ : dom $V \rightarrow Y$ is a function such that the relation $g \subset U$. A slice-contraction of $V$ onto $g$ in $U$ is a homotopy $\phi: V \times I \rightarrow U$ such that $\phi_{0}=$ inclusion $V \rightarrow U$, $\phi((V \mid x) \times I) \subset U \mid x$ for any $x \in \operatorname{dom} V$, and $\phi_{1}(V \mid x)=g \mid x$. If there is a slice contraction of $V$ onto $g$ in $U$, we say that $V$ slice-contracts or is slice-contractible onto $g$ in $U$. A relation $R: X \rightarrow Y$ is slice-trivial in $X \times Y$ provided each neighborhood $U$ of $R$ in $X \times Y$ contains a neighborhood $V$ of $R$ in $X \times Y$ such that $V$ slice-contracts in $U$. An ANR trivial extension of a proper onto map $f: X^{\prime} \rightarrow X$ is a proper onto map $f_{+}: X_{+}^{\prime} \rightarrow X_{+}$from an ANR $X_{+}^{\prime}$ to a metric space $X_{+}$to which are associated closed embeddings $i: X^{\prime} \rightarrow X_{+}^{\prime}$ and $j: X \rightarrow X_{+}$such that $f_{+} \circ i=j \circ f$ and $f_{+}$maps $X_{+}^{\prime}-i\left(X^{\prime}\right)$ homeomorphically onto $X_{+}-j(X)$. For a proper onto map $f: X^{\prime} \rightarrow X$ and a closed embedding $i: X^{\prime} \rightarrow X_{+}^{\prime}$ into an ANR $X_{+}^{\prime}$, there is an obvious ANR trivial extension $f_{+}: X_{+}^{\prime} \rightarrow X_{+}$as follows. Let $X_{+}=X_{+}^{\prime} \cup_{f \circ i-1} X$, the space obtained by attaching $X_{+}^{\prime}$ to $X$ via the map $f \circ i^{-1}: i\left(X^{\prime}\right) \rightarrow X$. Let $j: X \rightarrow$ $X_{+}$be the natural "inclusion," and let $f_{+}: X_{+}^{\prime} \rightarrow X_{+}$be the natural map such that $f_{+} \circ i=j \circ f$ and $f_{+}$is the identity on $X_{+}^{\prime}-i\left(X^{\prime}\right)$.

\section{Results}

Theorem 1. A cell-like map $f: X^{\prime} \rightarrow X$ is a hereditary shape equivalence if there exists a sequence $\left\{B_{n}\right\}_{n=1}^{\infty}$ of closed subsets of $X$ such that $N_{f} \subset \cup_{n=1}^{\infty} B_{n}$, $f \mid B_{n}^{\prime}: B_{n}^{\prime} \rightarrow B_{n}$ is a hereditary shape equivalence for each $n=1,2, \ldots$, and, 
for each $B_{n}$ and a neighborhood $U$ of $B_{n}$, there exists a neighborhood $V$ of $B_{n}$ such that $\frac{n}{V} \subset U$ and $\partial V$ intersects at most finitely many members of $\left\{B_{i}^{\prime}\right\}_{i=1}^{\infty}$. Proof. If we embed $X^{\prime}$ as a closed subset of ANR $X_{+}^{\prime}$, then the ANR trivial extension $f_{+}: X_{+}^{\prime} \rightarrow X_{+}$satisfies all the hypotheses in Theorem 1. Hence we may assume $X^{\prime}$ is an ANR.

According to G. Kozlowski [Ko, Theorems 5 and 10], it is enough to show that, for any open cover $\mathscr{M}$ of $X$, there exists a map $g: X \rightarrow X^{\prime}$ such that $g \circ f$ is $\mathscr{M}^{\prime}$-homotopic to the identity on $X^{\prime}$. We show it in five steps.

Let $\mathscr{M}$ be an open cover of $X$. Using results of F. D. Ancel's [An, A.2 and A.9], find a neighborhood $U_{0}$ of $f^{-1}$ in $X \times X^{\prime}$ such that $\left\{U_{0}(x) \mid x \in X\right\}$ refines $\mathscr{M}^{\prime}$.

Step 1. First we note that the implication (5) $\Rightarrow(3 \mathrm{~b})$ in [An, 4.5] applied to $f \mid B_{n}^{\prime}: B_{n}^{\prime} \rightarrow B_{n}$ shows that $f \mid B_{n}^{\prime}: B_{n}^{\prime} \rightarrow B_{n}$ is slice-trivial for each $n=$ $1,2, \ldots$. It easily follows from results of Ancel's that, for each $n=1,2, \ldots$, there exists an open cover $\mathscr{L}_{n}$ of $X$, a neighborhood $U_{n}$ of $f^{-1}$ in $X \times X^{\prime}$, and a neighborhood $M_{n}$ of $B_{n}$ such that

$$
\begin{gathered}
\operatorname{mesh} \mathscr{L}_{n}<1 / n, \quad \mathscr{L}_{n}^{\prime} \text { refines }\left\{U_{n-1}(x) \mid x \in X\right\}, \\
U_{n} \subset U_{n-1}, \quad\left\{U_{n}(x) \mid x \in X\right\} \text { refines } \mathscr{L}_{n}^{\prime}(\text { see }[\mathrm{An}, \mathrm{A} .9]), \\
U_{n} \mid M_{n} \text { slice-contracts in } U_{n-1} \quad(\text { see }[\mathrm{An}, 3.5]) .
\end{gathered}
$$

Step 2. By induction we will show that, for each $n=1,2, \ldots$, there are (possibly empty) open subsets $K_{n}$ and $H_{n}$ of $X$ and a slice-contraction $\chi^{n}:\left[\bigcup_{i=1}^{n}\left(U_{i} \mid \bar{K}_{i}\right)\right] \times I \rightarrow \bigcup_{i=1}^{n}\left(U_{i-1} \mid \bar{K}_{i}\right)$ such that

$$
\bar{K}_{n} \subset H_{n} \subset \bar{H}_{n} \subset M_{n}, \quad \bigcup_{i=1}^{n} B_{i} \subset \bigcup_{i=1}^{n} K_{i},
$$

and

$$
\bar{K}_{n} \text { intersects at most finitely many of }\left\{\bar{H}_{i}\right\}_{i=1}^{\infty} \text {, }
$$

As a first inductive step, we simply choose open subsets $K_{1}$ and $H_{1}$ of $X$ such that $B_{1} \subset K_{1} \subset \bar{K}_{1} \subset H_{1} \subset \bar{H}_{1} \subset M_{1}$ and $\partial K_{1}$ intersects at most finitely many of $\left\{B_{n}\right\}_{n=1}^{\infty}$. Certainly there is a slice-contraction $\chi^{1}:\left(U_{1} \mid \bar{K}_{1}\right) \times I \rightarrow$ $U_{0} \mid \bar{K}_{1}$.

Let $k>0$, and assume that, for each $n=1,2, \ldots, k$, there exist open subsets $H_{n}$ and $K_{n}$ of $X$ and a slice-contraction $\chi^{n}:\left[\bigcup_{i=1}^{n}\left(U_{i} \bar{K}_{i}\right)\right] \times I \rightarrow$ $\bigcup_{i=1}^{n}\left(U_{i-1} \bar{K}_{i}\right)$ satisfying (2.2) and
$\bar{K}_{n} \subset H_{n} \subset \bar{H}_{n} \subset M_{n}$,
$\bigcup_{i=1}^{n} B_{i} \subset \bigcup_{i=1}^{n} K_{i}, \partial K_{i}$ intersects at most finitely many of $\left\{B_{i}\right\}_{i=1}^{\infty}$, and $\bar{H}_{n} \cap \bar{K}_{i}=\varnothing$ if $B_{n} \cap$ $\partial K_{i}=\varnothing$ for $i<n$. 
If $B_{k+1} \subset \bigcup_{i=1}^{k} K_{i}$, then we simply choose $K_{k+1}=H_{k+1}=\varnothing$ and $\chi^{k+1}=\chi^{k}$. Otherwise, first we choose open subsets $H_{k+1}$ and $K_{k+1}$ of $X$ such that $\bar{K}_{k+1} \subset$ $H_{k+1} \subset \bar{H}_{k+1} \subset M_{k+1}, \bigcup_{i=1}^{k+1} B_{i} \subset \bigcup_{i=1}^{k+1} K_{i}, \bar{H}_{k+1} \cap \bar{K}_{i}=\varnothing$ if $\partial K_{i} \cap B_{k+1}=\varnothing$ for $i<k+1$, and $\partial K_{k+1}$ intersects at most finitely many of $\left\{B_{i}\right\}_{i=1}^{\infty}$. Then we define $\chi^{k+1}: \bigcup_{i=1}^{k+1}\left(U_{i} \mid \bar{K}_{i}\right) \times I \rightarrow \bigcup_{i=1}^{k+1}\left(U_{i-1} \mid \bar{K}_{i}\right)$ as follows.

$$
\chi^{k+1}(x, y, t)=\left\{\begin{array}{l}
\chi^{k}(x, y, t) \text { on }\left[\bigcup_{i=1}^{k+1}\left(U_{i} \mid \bar{K}_{i}\right)-\left(U_{k+1} \mid H_{k+1}\right)\right] \times I, \\
\chi^{k}(\psi(x, y, \lambda(x) \cdot t), \mu(x) \cdot t) \\
\text { on }\left\{\left[\bigcup_{i=1}^{k+1}\left(U_{i} \mid \bar{K}_{i}\right)\right] \cap\left[U_{k+1} \mid\left(\bar{H}_{k+1}-K_{k+1}\right)\right]\right\} \times I, \\
\psi(x, y, t) \text { on }\left[U_{k+1} \mid \bar{K}_{k+1}\right] \times I,
\end{array}\right.
$$

where $\psi$ is a slice-contraction of $U_{k+1} \mid \bar{H}_{k+1}$ in $U_{k} \mid \bar{H}_{k+1}$ and $\mu, \lambda: X \rightarrow[0,1]$ are maps such that $\lambda\left(X-H_{k+1}\right)=\{0\}, \mu\left(\bar{K}_{k+1}\right)=\{0\}$, and $\mu^{-1}(1) \cup \lambda^{-1}(1)=$ $X$. Then we easily see that, for each $n=1,2, \ldots, k+1,(2.1)^{\prime}$ and (2.2) are satisfied by $\left\{K_{n}\right\}_{n=1}^{k+1},\left\{H_{n}\right\}_{n=1}^{k+1}$, and $\left\{\chi^{n}\right\}_{n=1}^{k+1}$. Now we can easily claim (2.1) and (2.2).

Step 3. For each $n=1,2, \ldots$, we define a slice-contraction

$$
\phi^{n}:\left[\bigcup_{i=1}^{n}\left(U_{i} \mid \bar{K}_{i}\right)\right] \times I \rightarrow \bigcup_{i=1}^{n}\left(U_{i-1} \mid \bar{K}_{i}\right) \quad \text { as } \phi^{n}(x, y, t)=\chi^{n(0)}(x, y, t)
$$

where $n(0)$ is the smallest integer satisfying $\left(\bigcup_{i=1}^{n} \bar{K}_{i}\right) \cap \bar{H}_{m}=\varnothing$ for any $m>n(0)$. Using (2.1) and (2.2) we can show that each $\phi_{n}$ is well defined and continuous. Furthermore, $\phi_{n}=\phi_{n+1}$ on $\bigcup_{i=1}^{n}\left(U_{i} \mid \bar{K}_{i}\right) \times I$ for each $n=$ $1,2, \ldots$.

Step 4. Let $K=\bigcup_{i=1}^{\infty} K_{i}$ and $U=\bigcup_{i=1}^{\infty}\left(U_{i-1} \mid \bar{K}_{i}\right)$. By induction we will show that, for each $n=1,2, \ldots$, there exist a map $g_{n}: \bigcup_{i=1}^{n} \bar{K}_{i} \rightarrow X^{\prime}$ and a homotopy $F^{n}:\left(\bigcup_{i=1}^{n} \bar{K}_{i}\right)^{\prime} \times I \rightarrow X^{\prime}$ such that

$$
\begin{aligned}
& g_{n}=g_{n+1} \text { on } \bigcup_{i=1}^{n} \bar{K}_{i}, \quad g_{n}=f^{-1} \text { on }\left(\bigcup_{i=1}^{n} \bar{K}_{i}\right)-K, \\
& F_{0}^{n}=\text { inclusion }\left(\bigcup_{i=1}^{n} \bar{K}_{i}\right)^{\prime} \rightarrow X^{\prime}, \quad F_{1}^{n}=g_{n} \circ f \mid\left(\bigcup_{i=1}^{n} \bar{K}_{i}\right)^{\prime}, \\
& F^{n}(x, t)=x \text { for each }(x, t) \in\left[\left(\bigcup_{i=1}^{n} \bar{K}_{i}\right)^{\prime}-K^{\prime}\right] \times I \\
& F^{n}=F^{n+1} \text { on }\left(\bigcup_{i=1}^{n} \bar{K}_{i}\right)^{\prime} \times I, \\
& F^{n}(\{x\} \times I) \subset U(f(x)) \text { for any } x \in\left(\bigcup_{i=1}^{n} \bar{K}_{i}\right)^{\prime}
\end{aligned}
$$


Let $\phi^{n}$ slice-contract $\bigcup_{i=1}^{n} U_{i} \mid \bar{K}_{i}$ onto $g_{n}^{\prime}$ in $U$. Then it follows from a proposition of F. D. Ancel's [An, 2.1.(1)] that, for each $n=1,2, \ldots, g_{n}^{\prime}$ is continuous function. Furthermore, $g_{n}^{\prime}=g_{n+1}^{\prime}$ on $\bigcup_{i=1}^{n}\left(U_{i} \mid \bar{K}_{i}\right)$ for each $n=1,2, \ldots$. Let $\pi: X \times X^{\prime} \rightarrow X^{\prime}$ be the projection map. Notice that $f^{-1} \mid\left(\bar{K}_{1}-K\right): \bar{K}_{1}-K \rightarrow X^{\prime}$ is a continuous map and that $G^{1}:\left(\bar{K}_{1}-K\right) \times I \rightarrow X^{\prime}$ defined by $G^{1}(x, t)=\pi \circ \phi^{-1}\left(x, f^{-1}(x), t\right)$ is a homotopy from $G_{0}^{1}=f^{-1} \mid\left(\bar{K}_{1}-\right.$ $K)$ to $G_{1}^{1}=g_{1}^{\prime} \mid\left(\bar{K}_{1}-K\right)$ such that $G^{1}(\{x\} \times I) \subset U(x)$ for any $x \in \bar{K}_{1}-K$. By modifying the homotopy extension property of an ANR $X^{\prime}$, we obtain an extension $\bar{G}^{1}: \bar{K}_{1} \times I \rightarrow X^{\prime}$ of $G^{1}$ such that $\bar{G}^{1}(\{x\} \times I) \subset U(x)$ for each $x \in \bar{K}_{1}$. Define $g_{1}: \bar{K}_{1} \rightarrow X^{\prime}$ as $g_{1}(x)=\bar{G}_{1}(x, 0)$. Let

$$
P_{1}=\left[\left(\bar{K}_{1}-K\right)^{\prime} \times I \times I\right] \cup\left[\left(\bar{K}_{1}\right)^{\prime} \times\{0,1\} \times I\right] \cup\left[\left(\bar{K}_{1}\right)^{\prime} \times I \times\{1\}\right],
$$

and define $\Lambda_{1}: P_{1} \rightarrow X^{\prime}$ as

$$
\Lambda_{1}(x, s, t)= \begin{cases}\pi \circ \phi^{1}(f(x), x, s t) & \text { on }\left(\bar{K}_{1}-K\right)^{\prime} \times I \times I, \\ x & \text { on }\left(\bar{K}_{1}\right)^{\prime} \times\{0\} \times I, \\ \bar{G}^{1}(f(x), t) & \text { on }\left(\bar{K}_{1}\right)^{\prime} \times\{1\} \times I, \\ \pi \circ \phi^{1}(f(x), x, s) & \text { on }\left(\bar{K}_{1}\right)^{\prime} \times I \times\{1\} .\end{cases}
$$

Then we can easily check that $\Lambda_{1}$ is a well defined continuous function. By modifying the homotopy extension property of an ANR $X^{\prime}$, we get an extension $\bar{\Lambda}_{1}:\left(\bar{K}_{1}\right)^{\prime} \times I \times I \rightarrow X^{\prime}$ of $\Lambda_{1}$ such that $\bar{\Lambda}_{1}(\{x\} \times I \times I) \subset U(f(x))$ for each $x \in\left(\bar{K}_{1}\right)^{\prime}$. Now we define $F^{1}:\left(\bar{K}_{1}\right)^{\prime} \times I \rightarrow X^{\prime}$ as $F^{1}(x, s)=\bar{\Lambda}_{1}(x, s, 0)$.

We will show how to get $g_{2}$ and $F^{2}$. First we notice that $g_{1} \cup f^{-1} \mid\left(\bar{K}_{2}-K\right)$ defined a continuous function from $\bar{K}_{1} \cup\left(\bar{K}_{2}-K\right)$ to $X^{\prime}$. Define $G^{2}:\left[\bar{K}_{1} \cup\right.$ $\left.\left(\bar{K}_{2}-K\right)\right] \times I \rightarrow X^{\prime}$ and $G^{2}=\bar{G}^{1}$ on $\bar{K}_{1} \times I$ and $G^{2}(x, t)=\pi \circ \phi^{2}\left(x, f^{-1}(x), t\right)$ for any $(x, t) \in\left(\bar{K}_{2}-K\right) \times I$. Then $G^{2}$ is a homotopy from $g_{1} \cup f^{-1} \mid\left(\bar{K}_{2}-K\right)$ to $g_{2}^{\prime}$ such that $G^{2}(\{x\} \times I) \subset U(x)$ for any $x \in \bar{K}_{1} \cup\left(\bar{K}_{2}-K\right)$. By modifying the homotopy exntesion property of an ANR, we can find an extension $\bar{G}^{2}:\left(\bar{K}_{1} \cup\right.$ $\left.\bar{K}_{2}\right) \times I \rightarrow X^{\prime}$ of $G^{2}$ such that $\bar{G}^{2}(\{x\} \times I) \subset U(x)$ for each $x \in \bar{K}_{1} \cup \bar{K}_{2}$. Define $g_{2}: \bar{K}_{1} \cup \bar{K}_{2} \rightarrow X^{\prime}$ as $g_{2}(x)=\bar{G}^{2}(x, 0)$. Then $g_{2}$ is an extension of $g_{1}$ and $g_{2}=f^{-1}$ on $\left(\bar{K}_{1} \cup \bar{K}_{2}\right)-K$. Let

$$
\begin{aligned}
P_{2}=\left\{\left[\bar{K}_{1} \cup\left(\bar{K}_{2}-K\right)\right]^{\prime} \times I \times I\right\} \cup\left\{\left(\bar{K}_{1} \cup \bar{K}_{2}\right)^{\prime} \times\right. & \{0,1\} \times I\} \\
& \cup\left\{\left(\bar{K}_{1} \cup \bar{K}_{2}\right)^{\prime} \times I \times\{1\}\right\},
\end{aligned}
$$


and define $\Lambda_{2}: P_{2} \rightarrow X^{\prime}$ as

$$
\Lambda_{2}(x, s, t)= \begin{cases}\pi \circ \phi^{2}(f(x), x, s t) & \text { on }\left(\bar{K}_{2}-K\right)^{\prime} \times I \times I, \\ x & \text { on }\left(\bar{K}_{2}\right)^{\prime} \times\{0\} \times I, \\ \bar{G}^{2}(f(x), t) & \text { on }\left(\bar{K}_{2}\right)^{\prime} \times\{1\} \times I, \\ \pi \circ \phi^{2}(f(x), x, s) & \text { on }\left(\bar{K}_{2}\right)^{\prime} \times I \times\{1\}, \\ \bar{\Lambda}_{1}(x, s, t) & \text { on }\left(\bar{K}_{1}\right)^{\prime} \times I \times I .\end{cases}
$$

Then we easily see that $\Lambda_{2}$ is an extension of $\bar{\Lambda}_{1}$. By modifying the homotopy extension property of an ANR $X^{\prime}$, we extend $\Lambda_{2}$ to $\bar{\Lambda}_{2}:\left(\bar{K}_{1} \cup \bar{K}_{2}\right)^{\prime} \times I \times I \rightarrow X^{\prime}$ such that $\bar{\Lambda}_{2}(\{x\} \times I \times I) \subset U(f(x))$ for any $x \in\left(\bar{K}_{1} \cup \bar{K}_{2}\right)^{\prime}$. Now define $F^{2}:\left(\bar{K}_{1} \cup \bar{K}_{2}\right)^{\prime} \times I \rightarrow X^{\prime}$ as $F^{2}(x, s)=\bar{\Lambda}_{2}(x, s, 0)$. We easily see that $F^{2}$ is an extension of $F^{1}, F_{0}^{2}=$ inclusion, and $F_{1}^{2}=g_{2} \circ f \mid\left(\bar{K}_{1} \cup \bar{K}_{2}\right)^{\prime}$. Furthermore, $F^{2}(\{x\} \times I) \subset U(f(x))$ for any $x \in\left(\bar{K}_{1} \cup \bar{K}_{2}\right)^{\prime}$ and $F^{2}(x, t)=x$ for any $(x, t) \in\left[\left(\bar{K}_{1} \cup \bar{K}_{2}\right)^{\prime}-K^{\prime}\right] \times I$.

Now, by induction, we claim that, for each $n=1,2, \ldots$, there exist a map $g_{n}: \bigcup_{i=1}^{n} \bar{K}_{i} \rightarrow X^{\prime}$ and a homotopy $F^{n}:\left(\bigcup_{i=1}^{n} \bar{K}_{i}\right)^{\prime} \times I \rightarrow X^{\prime}$ satisfying (4.1) and (4.2).

Step 5. Finally we define $g: X \rightarrow X^{\prime}$ as

$$
g(x)= \begin{cases}g_{n}(x) & \text { if } x \in K_{n} \text { for some } n=1,2, \ldots, \\ f^{-1}(x) & \text { if } x \in X-\bigcup_{i=1}^{\infty} K_{i}\end{cases}
$$

and define $F: X^{\prime} \times I \rightarrow X^{\prime}$ as

$$
F(x, t)= \begin{cases}F^{n}(x, t) & \text { if }(x, t) \in\left(K_{n}\right)^{\prime} \times I \text { for some } n=1,2, \ldots, \\ x & \text { if }(x, t) \in\left[X^{\prime}-\left(\bigcup_{i=1}^{\infty} K_{i}\right)^{\prime}\right] \times I .\end{cases}
$$

We will show $g$ is continuous. First notice that it is enough to show that $\left\{g\left(x_{n}\right)\right\}_{n=1}^{\infty}$ converges to $f^{-1}(x)$ for any sequence $\left\{x_{n}\right\}_{n=1}^{\infty}$ in $K$ converging to $x$ in $\partial K$. Since $g_{n}=f^{-1}$ on $\bigcup_{i=1}^{n} \bar{K}_{i}-K$ and $\bar{K}_{n}$ intersects at most finitely many of $\left\{\bar{K}_{i}\right\}_{i=1}^{\infty}$, it is enough to show that $\left\{g\left(x_{n}\right)=g_{n}\left(x_{n}\right)\right\}_{n=1}^{\infty}$ converges to $f^{-1}(x)$ for any sequence $\left\{x_{n}\right\}_{n=1}^{\infty}$ with $x_{n} \in K_{n}-\bigcup_{i=1}^{n-1} K_{i}$ converging to $x$ in $\partial K$. Consider a neighborhood $B\left(f^{-1}(x) ; \varepsilon\right)=\left\{y \in X^{\prime} \mid d\left(f^{-1}(x), y\right)<\varepsilon\right\}$ for any $\varepsilon>0$. Since $f$ is proper, there exists a neighborhood $B(x ; \delta)=$ $\{y \in X \mid d(x, y)<\delta\}$ of $x$ in $X$ for some $\delta>0$ such that $f^{-1}(B(x ; \delta)) \subset$ $B\left(f^{-1}(x) ; \varepsilon\right)$. Choose an integer $N$ such that $x_{n} \in B(x ; \delta / 2)$ for any $n \geq N$ and $1 / N<\delta / 2$. Then, for any $n \geq N, U\left(x_{n}\right) \subset f^{-1}(L)$ for some $L \in \mathscr{L}_{n}$. Since $f^{-1}\left(x_{n}\right) \cup g\left(x_{n}\right) \subset U\left(x_{n}\right)$, we have $\left\{x_{n}, f \circ g\left(x_{n}\right)\right\} \subset L$ and $\operatorname{diam} L<\delta / 2$ for any $n \geq N$. Therefore, for any $n \geq N$,

$$
d\left(f \circ g\left(x_{n}\right), x\right) \leq d\left(f \circ g\left(x_{n}\right), x_{n}\right)+d\left(x_{n}, x\right)<\delta / 2+\delta / 2=\delta .
$$

Hence $f \circ g\left(x_{n}\right) \in B(x, \delta)$, and $g\left(x_{n}\right) \in B\left(f^{-1}(x) ; \varepsilon\right)$ for $n \geq N$. Therefore $g$ is continuous. 
The continuity of $F$ can be checked by the same analysis that established the continuity of $g$. Finally we claim that $F$ is a $\mathscr{M}^{\prime}$-homotopy from the identity on $X^{\prime}$ to $g \circ f$ by noticing that $F(\{x\} \times I) \subset U(f(x)) \subset U_{0}(f(x))$ and that $\left\{U_{0}(x) \mid x \in X\right\}$ refines $\mathscr{M}^{\prime}$. Therefore $f$ is a hereditary shape equivalence.

As an immediate corollary of Theorem 1 we have the following.

Corollary 1. A cell-like map $f: X^{\prime} \rightarrow X$ is a hereditary shape equivalence if there exists a sequence $\left\{B_{n}\right\}_{n=1}^{\infty}$ of closed subsets of $X$ such that $N_{f} \subset \bigcup_{n=1}^{\infty} B_{n}$, $f \mid B_{n}^{\prime}$ is a hereditary shape equivalence for any $B_{n}$, and each $B_{n}$ has arbitrary small neighborhoods whose boundaries miss $\bigcup_{n=1}^{\infty} B_{i}$.

Some interesting cases of Corollary 1 follow.

Corollary 2 (G. Kozlowski [Ko]). A cell-like map $f: X^{\prime} \rightarrow X$ is a hereditary shape equivalence if there exists a sequence $\left\{B_{n}\right\}_{n=1}^{\infty}$ of closed subsets of $X$ such that $N_{f} \subset \cup_{n=1}^{\infty} B_{n}, f \mid B_{n}^{\prime}$ is a hereditary shape equivalence for each $B_{n}$, and $\left\{B_{n}^{\prime}\right\}_{n=1}^{\infty}$ forms a pairwise disjoint null-sequence.

Corollary 3. A cell-like map $f: X^{\prime} \rightarrow X$ is a hereditary shape equivalence if there exists a sequence $\left\{B_{n}\right\}_{n=1}^{\infty}$ of closed subsets of $X$ such that $N_{f} \subset \bigcup_{n=1}^{\infty} B_{n}, f \mid B_{n}^{\prime}$ is a hereditary shape equivalence for each $B_{n}$, and $\left\{B_{n}\right\}_{n=1}^{\infty}$ forms a pairwise disjoint null-sequence.

Using a theorem of L. Tumarkin's [Na, Theorem II.10, p. 32], a theorem of F. D. Ancel's [An, Theorem 5.1], and Corollary 1, we extend Corollary 1.

Theorem 2. A cell-like map $f: X^{\prime} \rightarrow X$ from a compactum onto a metric space $X$ is a hereditary shape equivalence if there exist a sequence $\left\{B_{n}\right\}_{n=1}^{\infty}$ of closed subsets of $X$ and an integer $k$ such that $N_{f} \subset \bigcup_{n=1}^{\infty} B_{n}, f \mid B_{n}^{\prime}$ is a hereditary shape equivalence for each $B_{n}$, and, for each $B_{n}$ and a neighborhood $U$ of $B_{n}$, there exists a neighborhood $V$ of $B_{n}$ such that $\bar{V} \subset U$ and $\operatorname{dim}[\partial V \cap$ $\left.\left(\bigcup_{i=1}^{\infty} B_{i}\right)\right] \leq k$.

Proof. For each $B_{n}$ and each integer $m>0$, choose a neighborhood $V_{n, m}$ of $B_{n}$ such that $\operatorname{dim}\left[\partial V_{n, m} \cap\left(\bigcup_{i=1}^{\infty} B_{i}\right)\right] \leq k$ and $\bar{V}_{n, m} \subset\left\{x \in X \mid d\left(B_{n}, x\right)<\right.$ $1 / m\}$, and let $K=\bigcup_{n=1}^{\infty} \bigcup_{m=1}^{\infty}\left[\partial V_{n, m} \cap\left(\bigcup_{i=1}^{\infty} B_{i}\right)\right]$. Notice that $\operatorname{dim} K \leq k$. By a theorem of L. Tumarkin's [Na, Theorem II.10, p. 32], we find a $G_{\delta}$-subset $L$ of $X$ such that $\operatorname{dim} L \leq k, K \subset L$, and $X-L=\bigcup_{i=1}^{\infty} C_{i}$ for closed subsets $C_{i}$ 's of $X$. By a theorem of F. D. Ancel's [An, Theorem 5.1], to show $f$ is a hereditary shape equivalence, it is enough to show that $f \mid C_{i}^{\prime}: C_{i}^{\prime} \rightarrow C_{i}$ is a hereditary shape equivalence for each $i=1,2, \ldots$. It is not hard to see that $f \mid C_{i}^{\prime}: C_{i}^{\prime} \rightarrow C_{i}$ and the sequence $\left\{B_{n} \cap C_{i}\right\}_{n=1}^{\infty}$ of closed subsets of $C_{i}$ satisfy the hypothesis in Corollary 1 . Therefore each $f \mid C_{i}^{\prime}$ is a hereditary shape equivalence, hence $f$ is a hereditary shape equivalence. 


\section{QUESTIONS}

Recall the definition of a strong transfinite dimension, Ind, which is given inductively (see [Na]). Ind $X=-1$ provided $X$ is the empty space. Ind $X \leq \alpha$ for an ordinal $\alpha$ provided for each closed subset $A$ of $X$ and a neighborhood $U$ of $A$ there exists a neighborhood $V$ of $A$ such that $V \subset U$ and $\operatorname{Ind}(\partial V) \leq$ $\beta$ for some ordinal $\beta<\alpha$.

Now we raise two questions which can be shown equivalent to each other by adopting the analyses in the proof of Theorem 2 and by using a theorem of $F$. D. Ancel's [An] which states that a strong transfinite dimensional subset of a metric space is contained in a $G_{\delta}$-subset which is countable dimensional.

Question 1. Is a cell-like map a hereditary shape equivalence if the nondegeneracy set is contained in the countable union of pairwise disjoint finite dimensional closed subsets?

Question 2. Is a cell-like map $f: X^{\prime} \rightarrow X$ a hereditary shape equivalence if there exists a sequence $\left\{B_{n}\right\}_{n=1}^{\infty}$ of finite dimensional closed subsets of $X$ such that $N_{f} \subset \bigcup_{n=1}^{\infty} B_{n}$ and $\bigcup_{n \neq m}\left(B_{n} \cap B_{m}\right)$ has a strong transfinite dimension?

\section{REFERENCES}

[An] F. D. Ancel, The role of countable dimensionality in the theory of cell-like relations, Trans. Amer. Math. Soc. 287 (1985), 1-40.

[Du] J. Dugundji, Topology, Allyn and Bacon, Boston, 1966.

[DW] R. J. Daverman and J. J. Walsh, Examples of cell-like maps that are not shape equivalences, Michigan Math. J. 30 (1983), 17-30.

[Ha] W. E. Haver, Mappings between ANR's that are fine homotopy equivalences, Pacific J. Math. 58 (1975), 457-461.

[Hu] S. T. Hu, Theory of retracts, Wayne State Univ. Press, Detroit, MI, 1965.

[Ko] G. Kozlowski, Images of ANR's, Trans. Amer. Math. Soc. (to appear).

[La] R. A. Lacher, Cell-like mappings. I, Pacific J. Math. 30 (1969), 717-731.

[MR] J. Mogilski and H. Roslaniec, Cell-like decompositions of ANR's, Bull. Acad. Polon. Sci. Ser. Sci. Math. Astronom. Phys. (to appear).

[Na] J. Nagata, Modern dimension theory, Wiley, New York, 1965.

[Sh] R. B. Sher, Realizing cell-like maps in Euclidean space, General Topology Appl. 2 (1972), 75-89.

[Ta] J. L. Taylor, A counterexample in shape theory, Bull. Amer. Math. Soc. 81 (1975), 629-632.

Department of Mathematics, Tennessee Technological University, Cookeville, TenNESSEE 38501 